\title{
No difference in survivorship after unicompartmental knee arthroplasty with or without an intact anterior cruciate ligament
}

Adam Boissonneault ${ }^{1}$, Hemant Pandit ${ }^{1,2}$, Elise Pegg ${ }^{1}$, Cathy Jenkins ${ }^{2}$, Harinderjit Singh Gill ${ }^{1}$, Christopher A. F. Dodd ${ }^{2}$, Christopher L. M. H. Gibbons ${ }^{2}$, David W. Murray ${ }^{1,2}$

[1] Nuffield Department of Orthopaedics, Rheumatology and Musculoskeletal Sciences (NDORMS), University of Oxford, Headington, Oxford, OX3 7LD, UK

[2] Nuffield Orthopaedic Centre (NOC), University of Oxford, Headington, Oxford, OX3 7LD, UK

\begin{abstract}
Purpose

Anterior cruciate ligament deficiency (ACLD) has been considered a contraindication for Oxford unicompartmental knee arthroplasty (UKA) because of the reported higher incidence of failure when implanted in ACLD knees. However, given the potential advantages of UKA over total knee arthroplasty (TKA), we have performed UKA in a limited number of patients with ACL-deficiency and end-stage medial compartment osteoarthritis (OA) over the past 11 years. The primary aim of this study was to establish the clinical outcome of this cohort; the secondary aim was to compare both clinical and radiographic data with a matched cohort of ACL-intact (ACLI) patients who have undergone UKA for anteromedial OA.
\end{abstract}

\section{Methods}

This retrospective observational study describes the clinical and radiological outcome in 46 medial Oxford UKAs implanted in 42 consecutive patients with ACL deficiency and concomitant symptomatic medial compartment OA at mean follow up of 5 years. It also compares the outcomes with a matched cohort of UKA patients with an intact ACL (ACLI group).

Results

At the time of last follow up, there was no significant difference in clinical results or survivorship between the two groups in this study.

Conclusion

The successful short-term results of the ACLD group suggest ACL-deficiency may not always be a contraindication to Oxford UKA as previously thought. Until longer-term data is available, however, we maintain our recommendation that ACLD be considered a contraindication.

Level of Evidence

III.

Keywords - Unicompartmental Knee Arthroplasty, Anterior Cruciate Ligament Deficiency 


\section{Introduction}

There are mixed views on whether a functionally intact anterior cruciate ligament (ACL) is a necessary prerequisite for successful unicompartmental knee arthroplasty (UKA) [6]. While increased failure rates associated with ACL deficiency (ACLD) have been shown for both fixed-bearing UKAs [5] and mobile-bearing UKAs [7], others report no increased incidence of complication attributable to $\operatorname{ALCD}[2,3]$. The most common reported reason for UKA failure in ACLD knees is aseptic tibial component loosening.

ACLD is currently a contraindication for patients undergoing UKA with the Oxford Partial Knee (Biomet, Bridgend, UK) mobile-bearing prosthesis. The contraindication is based on the reported higher incidence of failure when implanted in ACLD knees [7]. In the original reported series, 6 of the 28 cases failed (21.4\%) within the first two years of implantation. Subsequent to these findings, White et al. [19] described the underlying pathophysiology of OA progression in ACLD knees, which further supported the view that UKA is not advisable in such patients.

Anteromedial osteoarthritis was determined to be a disease specific to ACL-intact knees and the primary indication for UKA [19]. Without a functional ACL, the tibial erosion extends posteriorly so the pattern of the disease is posteromedial $[8,13-14]$. This is associated with a fixed varus deformity and increased degeneration of the lateral compartment, both of which are considered to be incompatible with UKA. It is worth noting that these pathological changes are associated with patients having primary osteoarthritis with secondary ACL injury. When ACL trauma or rupture is the primary pathology, which is generally the case for younger patients with concomitant knee OA and ACLD, the pattern of OA differs [16] and our current routine management for young ACLD patients is a combined ACL reconstruction with UKA.

Since the original series of Oxford medial UKAs showed an increased incidence of failure in ACLD knees, the designer surgeons have mainly used the implant in treatment of end-stage anteromedial osteoarthritis for knees with a functionally intact ACL. However, due to the potential advantages of UKA over TKA - increased mobility, improved function, better restoration of natural biomechanics, reduced mortality and morbidity, and shorter hospital stay [15] - we have performed UKA in ACLD knees in a limited number of cases, mostly at the request of the patient. Such patients were aware of the increased risk of failure.

If ACLD is indeed a causative agent of aseptic tibial component loosening, we expect to see a failure rate in our ACLD cohort similar to that in the original series. Therefore, the primary aim of this study was to establish the survivorship and clinical outcome of this cohort; the secondary aim was to compare both clinical and radiographic data with a matched cohort of ACL-intact (ACLI) patients who have undergone UKA for anteromedial OA. 


\section{Materials and Methods}

Since the phase 3 Oxford UKA was introduced in 1998, our patients have been prospectively followed by an independent physiotherapist. The data collected include patient demographics, intra-operative findings, clinical scores, complications and revisions. Among the operative findings are information on the state of the ACL and that of the articular cartilage within the knee joint.

This study reports on 46 medial UKAs implanted in 42 consecutive patients with ACL deficiency and concomitant symptomatic medial compartment osteoarthritis that underwent minimally invasive phase 3 Oxford UKA under the care of three surgeons between January 2001 and June 2011. Patients were categorized as ACL-deficient and included in this study only if intra-operative findings determined the ACL was not functionally intact - either friable/fragmented or completely absent (Table I).

Each patient in the ACLD group was matched for age, gender and follow-up time with a randomly selected patient who had undergone UKA for end-stage osteoarthritis with an intact ACL (Table II). On average, patients were matched for age within 0.5 years of one another and for follow-up time within 1.0 years of one another (up to two years deviation was accepted). Patients were matched exactly for gender. All knees in the control group were categorised intra-operatively as having normal ACLs according to our ACL assessment guidelines.

ACL assessment. During surgery, the surgeon assesses the integrity of the ACL by visual inspection as well as by pulling on it with a tendon hook. The ACL is then categorised as normal, with synovial damage, longitudinal splits, friable and fragmented, or absent. This method of ACL assessment is utilised for several reasons. In arthritic knees, the variation in size of tibial erosion and/or presence of macroscopic bone deformities, osteophytes and soft tissue contracture, make it quite difficult to accurately assess the integrity of the ACL by routine clinical tests. In addition, presence of intercondylar notch osteophytes can obscure the visualisation of the ACL on MRI scans. Therefore, we have always relied on intra-operative assessment (under direct vision) as a preferred option.

Articular cartilage assessment. The articular cartilage of the lateral (or unaffected) femoral condyle is categorised as normal, with superficial damage, partial thickness cartilage loss (PTCL), focal $\left(<2 \mathrm{~cm}^{2}\right)$ full thickness cartilage loss (FTCL), or extensive $\left(>2 \mathrm{~cm}^{2}\right)$ FTCL. A further note about the location of damage to the articular cartilage is also made this indicates whether the damage is localised to the weight bearing central portion, the non-weight bearing portion by the intercondylar notch, or both. 
Clinical assessment: The instruments for clinical assessment were the Oxford Knee Score (OKS) [4], a validated patient questionnaire $(0=$ worst outcome, 48 = best outcome), the American Knee Society Score (both objective and functional) [9], and the Tegner Activity Score [17]. All clinical data and associated follow-up were collected by an independent physiotherapist.

Pre-operative radiological assessment: Lateral radiographs were analysed to determine the posterior extent of cartilage and bone erosion on the medial tibial plateau. Previously described methods were used to distinguish the medial tibial plateau from the lateral [10], and to classify the erosion [12]. A custom Matlab (Mathworks Inc., Natick, MA, USA) program was used to measure the native tibial slope on lateral radiographs. The tibial slope was the angle between a line drawn through the anterior and posterior margins of the native tibial plateau, and a perpendicular line to the tibial axis. The tibial axis was defined by a line drawn along the posterior border of the diaphysis of the tibia.

The same software was used to determine the degree of varus angular deformity in weight-bearing, anteroposterior radiographs. The angular deformity was the angle between the tibial axis, defined by a line drawn through the midpoint of the proximal tibia and distal tibia, and the femoral axis, defined by a line drawn through the midpoint of the proximal femur and distal femur.

Post-operative radiological assessment: A custom Matlab program was used to measure the posterior slope of the tibial implant on lateral radiographs. The posterior slope of the tibial implant was the angle between a line drawn through the anterior and posterior margins of the tibial plate of the implant, and a perpendicular line to the tibial axis.

The same software was used to determine the post-operative tibiofemoral angle in anteroposterior radiographs. The postoperative tibiofemoral angle was determined in the same manner as the pre-operative angular deformity, as previously described.

All radiographs, both pre-and post-operative, were assessed by an independent investigator. Using the custom software, the same independent investigator analysed a series of 30 radiographs at 3 separate time intervals to determine an intraclass correlation of 0.97 . The same series of radiographs was then analysed by a separate investigator and the interobservor error was found to be 0.93 . 
Radiographs at the time of last follow-up were assessed for evidence of implant loosening (subsidence and/or presence of radiolucency). If a radiolucency was present, it was classified as physiological or pathological based on previous descriptions [18].

Statistical analysis: PASW statistical software (SPSS, Chicago, IL, USA, version 18) was used to analyse clinical and radiological data. MATLAB software (2009) was used to analyse survivorship via KMPLOT: Kaplan-Meier estimation of the survival function [1].

\section{Results}

Clinical assessment: All clinical scores for both groups were found to improve post-operatively (Table III). The ACLD group had a significantly lower pre-operative objective American Knee Society Score (AKSS) than the ACLI group $(\mathrm{p}=$ 0.001); there was no significant difference in other pre-operative clinical scores. The lower pre-operative objective AKSS score for the ACLD group was not seen to correlate to pre-operative varus deformity or any other pre-operative assessments. There was no significant difference in any of the clinical scores at 1- or 5-year follow-up between the two groups.

Patients with an absent ACL had a median pre-operative OKS of 25 (18 to 33) and 1-year follow-up OKS of 41 (25 to 48). Patients with a friable/fragmented ACL had a median pre-operative OKS of 27 (13 to 39) and 1-year follow-up OKS of 44 (21 to 48). Whether patients had friable/fragmented, absent or normal ACLs, there was no significant difference between pre-operative OKS scores, 1-year follow-up OKS scores, or 5-year follow-up OKS scores. This confirms the data for the ACLD group as a whole are not skewed by the two sub-populations we defined as having non-functional ACLs.

Lateral compartment: The non-weight bearing medial side of the lateral femoral condyle was determined to be either normal or having superficial damage in $93 \%$ of the knees in the ACLI group, but for only $50 \%$ of the knees in the ACLD group (Table IV). While the ACLI group had significantly more knees with normal lateral compartments, the ACLD group had significantly more knees with lateral compartments that contained partial thickness cartilage loss or focal full thickness cartilage loss. Only 3 knees (7\%) in the ACLI group had either PTCL or focal FTCL, while 23 knees (50\%) in the ACLD group had PTCL or focal FTCL. The focal FTCL in all cases was located on the medial aspect of the lateral femoral condyle. 
Tibial plateau: Analysis of pre-operative lateral radiographs revealed the tibial plateau defects in ACLI knees were all central and anterior, with no defects extending to the posterior joint margin. In contrast, the tibial erosions in ACLD knees were all central and posterior (Figure 1). The tibial defect reached the posterior joint margin in 12 of the 46 ACLD knees $(26 \%)$.

There was no significant difference in the pre-operative native tibial slope or the post-operative posterior slope of the tibial implant between the two groups (Table V.). The average posterior slope of the tibial implant was found to decrease from the average pre-operative slope for both groups.

\begin{abstract}
Alignment of knee joint: The average pre-operative varus angular deformity was significantly greater in the ACLD group than in the ACLI group (Table V). There were 12 knees in the ACLD group with a pre-operative varus angular deformity greater than $5.0^{\circ}\left(5.7^{\circ}\right.$ to $\left.14.1^{\circ}\right)$. Only 2 knees in the ACLI group had a pre-operative varus angular deformity greater than $5.0^{\circ}\left(6.2^{\circ}\right.$ to $\left.8.3^{\circ}\right)$.
\end{abstract}

While the average post-operative knee alignment was a valgus orientation for both groups, there was a significant difference in the post-operative tibiofemoral angle between the two groups. There were 8 knees in the ACLD group that retained a varus alignment $\left(0.1^{\circ}\right.$ to $\left.4.9^{\circ}\right)$ post-operatively, compared to only 2 knees $\left(0.0^{\circ}\right.$ to $\left.0.1^{\circ}\right)$ in the ACLI group.

Component loosening: No patient in either group had evidence of component subsidence or pathological radiolucency. There was no significant difference (n.s.) in the presence of physiological radiolucencies. There were six physiological radiolucencies in the ACLD group and all were partial: 3 around the keel, 2 medial to the keel, and 1 lateral to the keel. There were 3 radiolucencies in the ACLI group and all were partial: 2 around the keel and 1 medial to the keel.

Complications: There was one patient in the ACLD group (friable/fragmented) who developed lateral compartment arthritis and subsequently underwent revision to a TKR. The patient had pre-operative PTCL damage to the medial aspect of the lateral femoral condyle, and a post-operative tibiofemoral angle of $0.02^{\circ}$ of valgus. The failure occurred 3.5 years after implantation. No patient in the ACLI group had a significant complication.

\title{
Discussion
}

The most important finding of the present study was there was no significant difference (n.s.) between survival rates for UKAs implanted in ACL-deficient versus ACL-intact knees. The 5-year survival rate for medial UKAs in ACLD knees 
was $94 \%$ (95\% CI 90 to 100) with 19 patients at risk. In addition, the radiographic data and intra-operative findings presented here illustrate:

1. The extent of the tibial defect in the ACLD group extended more posteriorly, while in the ACLI group it remained centred centrally and anteriorly.

2. The ACLD group had significantly more degeneration in the non-weight bearing areas of the lateral compartment than the ACLI group.

3. The ACLD group achieved a significantly less amount of physiological valgus post-operatively, suggesting the ACLD group presented with medial collateral ligament contracture, or tibia vara.

The pathological changes seen in the ACLD group coincide with findings of previous literature [8, 13-14, 19], but does not seem to compromise results. Our results confirm previous literature that increased damage to the non-weight bearing medial side of the lateral compartment is not detrimental to clinical outcome results for UKA [11].

This study suggests a functionally intact anterior cruciate ligament is not an essential prerequisite for successful UKA. The revision rate of $2.2 \%$ for the ACLD group is significantly less $(\mathrm{p}=0.003)$ than the $21.4 \%$ reported in the original series of medial UKAs implanted in ACLD knees [7]. Where the most common method of failure in the original series was aseptic tibial component loosening, the only revision in this study was due to lateral compartment arthritis. In this case, the tibial component was found to be soundly fixed at the time of revision surgery.

The question that remains is why these results differ significantly from those presented in the original series of Oxford medial UKAs [7]. Some potential explanations include:

Evolution of surgical instruments and technique have made the fixation of the phase 3 Oxford procedure more reliable than the phase 1 procedure performed in the original series. The phase 3 procedure differs from the phase 1 procedure in that it allows ligament tensions to be balanced intra-operatively. A more accurate restoration of native muscle-tendon length may allow the hamstring muscles to function more optimally in stabilizing the tibia from subluxing anteriorly during heel strike.

Tightened indications and increased selection criteria for patients considered for UKA have filtered out poor candidates previously deemed acceptable. For example, 2 of the 6 failures in the original series correspond to simultaneous bilateral implantation on a patient with a pre-operative varus angular deformity of over $20^{\circ}$ in both knees, along with "severe anteroposterior instability [7]." Both of these implants failed within four months of operation. This example represents a patient we would not currently consider for UKA. To further distinguish the evolution of selection criteria, it is worth 
noting ACLD patients represented 37\% (28 of 75) of the total patient population receiving medial UKAs in the original series [7], whereas the 46 ACLD knees reported in this study represent less than $3 \%$ of the total patient population receiving UKAs in the same time period.

Patients in the original series had more severe or additional pathological changes which contributed to their failure. For example, they may have had appreciable damage to the central weight-bearing area of the lateral femoral condyle which was not present in the current series, they may have had gross rather than mild shortening of the MCL, or they may have had substantial rather than little sagittal instability.

Patients in the current series had a clinically insignificant amount of sagittal instability pre-operatively. It is possible patients in the current series had a negligible amount of sagittal instability pre-operatively or were not active enough post-operatively to test the stability of the implant against increased translational force. The latter is an unlikely blanket explanation, as one ACLD patient who underwent bilateral UKA (operations were one year apart) has remained an avid skier and reported 5-year OKS scores of 48 (max score) in both knees.

This study is limited in that it only provides short-term results. However, all the failures in the original series occurred within two years of implantation, and given 41 of 46 patients in this current series had a follow-up time over two years, similar failures should have been observed by this time if it were to be a problem. This study is also limited in that it only provides static radiological assessments and does not provide information regarding sagittal instability pre- or postoperatively. Future kinematic studies and longer-term clinical data are necessary to more completely assess the compatibility of ACL-deficiency with UKA. In addition, studies of ACLD patients with more severe disease need to be undertaken to more clearly define which patients with ACLD are appropriate for UKA and which are not.

\section{Conclusion}

As life expectancy continues to improve, there is a growing middle-aged and elderly population that are diagnosed with end-stage osteoarthritis but want to remain active. This study suggests Oxford UKA may present a new surgical treatment option for those among this population with concomitant ACL deficiency. However, while this study shows UKA can be successful in certain ACLD patients, we maintain our recommendation that patients considered for UKA should have a functionally intact ACL. This cautious approach is for two reasons: there is a lack of long-term results, and our current inability to define what distinguishes this ACLD cohort from the original series. Therefore we cannot offer guidelines as to which ACLD patients may be offered UKA at present. 


\section{Tables and Figures:}

Table I. Status of anterior cruciate ligament (ACL)

\begin{tabular}{lcc}
\hline & \multicolumn{2}{c}{ No. of knees } \\
\cline { 2 - 3 } Intra-operative findings & ACLD group* & ALCI group $^{\dagger}$ \\
\hline Normal & 0 & 46 \\
Friable/fragmented & 33 & 0 \\
Absent & 13 & 0 \\
Total & 46 & 46 \\
\hline${ }^{*}$ ACLD, anterior cruciate ligament deficiency & \\
${ }^{\dagger}$ ACLI, anterior cruciate ligament intact &
\end{tabular}

Table II. Patient demographics

\begin{tabular}{lll}
\hline & ACLD group $^{*}$ & ACLI group $^{\dagger}$ \\
\hline Mean age in years (SD) & $65(11)$ & $65(11)$ \\
Gender, M:F & $32: 10$ & $35: 10$ \\
Bilateral & 4 & 1 \\
Mean follow-up in years (SD) & $4.9(2.7)$ & $4.8(1.5)$ \\
\hline
\end{tabular}

Mean follow-up in years (SD) 4.9 (2.7)

*ACLD, anterior cruciate ligament deficiency

${ }^{\dagger} \mathrm{ACLI}$, anterior cruciate ligament intact

Note: no bilateral implants were simultaneous

Table III. Clinical outcome results

\begin{tabular}{|c|c|c|c|c|c|c|c|c|c|}
\hline \multirow[b]{2}{*}{ Assessment } & \multicolumn{3}{|c|}{ Pre-operative scores (median(range))* } & \multicolumn{3}{|c|}{ 1-year follow-up scores (median(range)) } & \multicolumn{3}{|c|}{ 5-year follow-up scores (median(range)) } \\
\hline & ACLD & ACLI & $\begin{array}{r}\text { p- } \\
\text { value }\end{array}$ & ACLD & ACLI & $\begin{array}{c}\text { p- } \\
\text { value }\end{array}$ & ACLD & ACLI & $\begin{array}{r}p- \\
\text { value }\end{array}$ \\
\hline $\mathrm{OKS}^{4}$ & 27 (13 to 39) & $29(9$ to 43$)$ & n.s. & $43(21$ to 48$)$ & $43(28$ to 48$)$ & n.s. & $43(20$ to 48$)$ & $44(20$ to 48$)$ & n.s. \\
\hline $\mathrm{AKSS}-\mathrm{O}^{9}$ & $42(15$ to 60$)$ & $54(25$ to 93$)$ & $\mathrm{p}=0.001$ & $85(53$ to 100$)$ & $85(50$ to 100$)$ & n.s. & $88(75$ to 90$)$ & $85(60$ to 98$)$ & n.s. \\
\hline AKSS $-\mathrm{F}^{9}$ & $70(45$ to 90$)$ & $70(40$ to 100$)$ & n.s. & $90(55$ to 100$)$ & $100(50$ to 100$)$ & n.s. & $100(40$ to 100$)$ & $73(40$ to 100$)$ & n.s. \\
\hline Tegner $^{17}$ & $3(1$ to 6$)$ & $2(0$ to 5$)$ & n.s. & $3(1$ to 6$)$ & $3(1$ to 6$)$ & n.s. & $2(1$ to 4$)$ & $2(1$ to 6$)$ & n.s. \\
\hline
\end{tabular}

* ACLD, anterior cruciate ligament deficiency; ACLI, anterior cruciate ligament intact; n.s., not significant

Table IV. State of lateral compartment

\begin{tabular}{lccr}
\hline & \multicolumn{2}{c}{ No. of knees } & \\
\cline { 2 - 3 } Uninvolved femur & ACLD & ALCI & p-value \\
\hline Normal & 14 & 27 & $\mathrm{p}=0.004$ \\
Superficial damage & 9 & 15 & n.s. \\
PTCL $^{\dagger}$ & 11 & 2 & $\mathrm{p}=0.006$ \\
Focal $\left(<2 \mathrm{~cm}^{2}\right) \mathrm{FTCL}^{\ddagger}$ & 12 & 1 & $\mathrm{p}=0.001$ \\
Extensive $\left(<2 \mathrm{~cm}^{2}\right) \mathrm{FTCL}^{\ddagger}$ & 0 & 0 & n.s. \\
\hline
\end{tabular}

$\uparrow$ Partial thickness cartilage loss

\$ Full thickness cartilage loss

NB: Data unavailable for one patient in ACLI group

Table V. Posterior tibial slope and knee alignment

\begin{tabular}{llcr}
\hline & \multicolumn{2}{c}{ Slope (mean (SD)) } & \\
\cline { 2 - 3 } & ACLD & ACLI & p-value \\
\hline Posterior tibial slope & & & \\
Pre-operative native tibia & $4.7^{\circ}\left(2.9^{\circ}\right)$ & $4.8^{\circ}\left(3.3^{\circ}\right)$ & n.s. \\
Post-operative tibial implant & $2.5^{\circ}\left(3.6^{\circ}\right)$ & $2.5^{\circ}\left(2.4^{\circ}\right)$ & n.s. \\
Knee alignment & & & \\
Pre-operative varus & $4.0^{\circ}\left(3.8^{\circ}\right)$ & $1.3^{\circ}\left(2.7^{\circ}\right)$ & $\mathrm{p}=0.006$ \\
Post-operative valgus & $2.4^{\mathrm{o}}\left(3.5^{\circ}\right)$ & $4.2^{\mathrm{o}}\left(3.0^{\circ}\right)$ & $\mathrm{p}=0.02$ \\
\hline
\end{tabular}



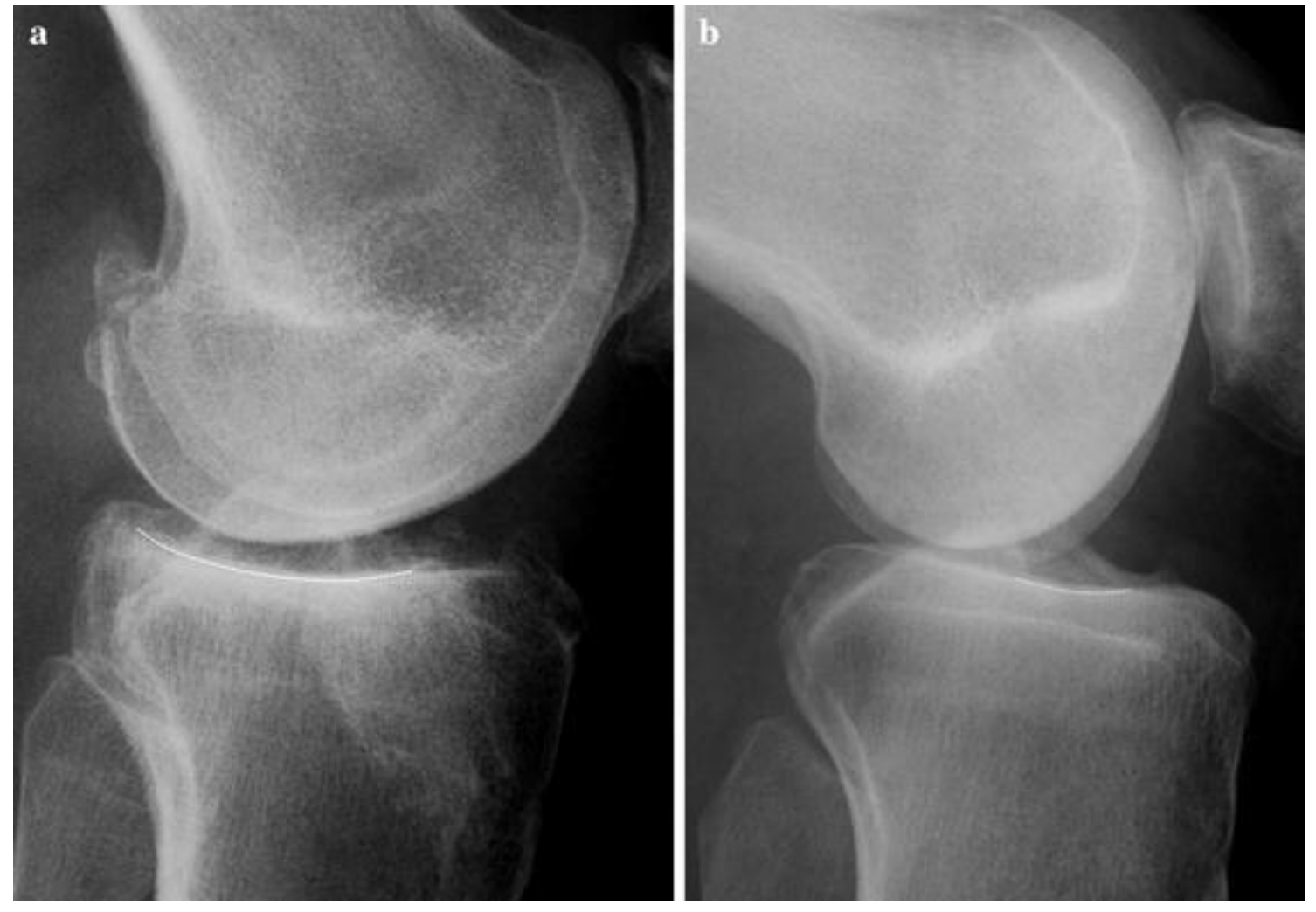

Fig. 1. (a) is a pre-operative lateral radiograph of an ACLD knee. The white dashed line represents the extent of the tibial defect, (b) is a pre-operative lateral radiograph of an ACLI knee. The white dashed line represents the extent of the tibial defect. In ACLD the erosion extends to the back; in ACLI it does not

\section{References:}

1. Cardillo G (2008) KMPLOT: Kaplan-Meier estimation of the survival function http://www.mathworks.com/matlabcentral/fileexchange/22293

2. Cartier P, Sanouiller JL, Greslamer RP (1996) Unicompartmental knee arthroplasty surgery. 10-year minimum followup period. J Arthroplasty 11:782-788

3. Christensen NO (1991) Unicompartmental prosthesis for gonarthrosis. A nine-year series of 575 knees from a Swedish hospital. Clin Orthop Relat Res 273:165-169

4. Dawson J, Fitzpatrick R, Murray DW, Carr AJ (1998) Questionnaire on the perceptions of patients about total knee arthroplasty. J Bone Joint Surg [Br] 80-B:63-69

5. Deschamps G, Lapeyre B (1987) Rupture of the anterior cruciate ligament: a frequently unrecognized cause of failure of unicompartmental knee prostheses. Apropos of a series of 79 Lotus prostheses with a follow-up of more than 5 years.

Rev Chir Orthop Reparatrice Appar Mot 73:544-551

6. Engh GA, Ammeen D (2004) Is an intact anterior cruciate ligmament needed in order to have a well-functioning unicondylar knee arthroplasty? Clin Orthop Relat Res 428:170-173 
7. Goodfellow JW, Kershaw CJ, D’A Benson MK, O’Connor JJ (1988) The Oxford knee for unicompartmental osteoarthritis: the first 103 cases. J Bone Joint Surg [Br] 70-B;692-701

8. Harmen MK, Markovich GD, Banks SA, Hodge WA (1998) Wear patterns on tibial plateaus from varus and valgus osteoarthritic knees. Clin Orthop Relat Res 352:149-158

9. Insall JN, Dorr LD, Scott RD, Scott WN (1989) Rationale of the Knee Society clinical rating system. Clin Orthop

Relat Res 248:13-14

10. Jacobsen K (1981) Gonylaxometry. Stress radiographic measurement of passive stability in the knee joints of normal subjects and patients with ligament injuries: accuracy and range of application. Acta Orthop Scand [Suppl] 52(194):1263

11. Kendrick BJ, Rout R, Bottomley NJ, Pandit H, Gill HS, Price AJ, Dodd CAF, Murray DW (2010) The implications of damage to the lateral femoral condyle on medial unicompartmental knee arthroplasty. J Bone Joint Surg [Br] 92B:374-379

12. Keyes GW, Carr AJ, Miller RK, Goodfellow JW (1992) The radiographic classification of medial gonarthrosis. Acta Orthop Scand 63(5):497-501

13. Lee G, Cushner FD, Vigoritta V, Scuderi GR, Insall JN, Scott NS (2005) Evaluation of the anterior cruciate ligament integrity and degenerative arthritic patterns in patients undergoing total knee arthroplasty. J Arthroplasty 20:59-65 14. Mullaji AB, Marawar SV, Luthra M (2008) Tibial articular cartilage wear in varus osteoarthritic knees: correlation with anterior cruciate ligament integrity and severity of deformity. J Arthroplasty 23:128-135

15. Murray, DW (2000) Unicompartmental knee arthroplasty: now or never? Orthopedics 23(9):979-980

16. Pandit H, Beard J, Jenkins C, Kimstra Y, Thomas NP, Dodd CAF, Murray DW (2006) Combined anterior cruciate reconstruction and Oxford unicompartmental knee arthoplasty. J Bone Joint Surg [Br] 88-B:887-892

17. Tegner Y, Lysholm J (1985) Rating systems in the evaluation of knee ligament injuries. Clin Orthop Relat Res $198: 43-49$

18. Tibrewal SB, Grant KA, Goodfellow JW (1984) The radiolucent line beneath the tibial components of the Oxford meniscal knee. J Bone Joint Surg [Br] 66-B:523-528

19. White SH, Ludkowski PF, Goodfellow JW (1991) Anteromedial osteoarthritis of the knee. J Bone Joint Surg [Br] 73 $\mathrm{B} ; 582-586$ 